\title{
The EOM: An Adaptive Energy Option, State and Assessment Model for Open Hybrid Energy Systems
}

\author{
Christian Derksen, Rainer Unland \\ DAWIS - University of Duisburg-Essen \\ Schützenbahn 70, 45127, Essen, Germany \\ Email: \{christian.derksen, rainer.unland@icb.uni-due.de\}@icb.uni-due.de
}

\begin{abstract}
The current transformation process of how energy is supplied attracts great interest from many different market players. As a consequence, many proprietary solutions for "smart" energy applications are flooding the market. This turns out to be rather a problem than part of the solution for the systematic development of future energy grids. Additionally, the absence of necessary standards blocks further developments that enable the creation of novel, market-driven and hybrid control solutions. To overcome these problems, we suggest a standardized control approach for hybrid energy systems by means of a so called Energy Option Model (EOM). This unifying model and the therewith developed decision support system provides the necessary technical understanding and the economic assessment options for network-connected energy conversion systems. Thus, it can be used for single onsite systems as well as for aggregated systems that are controlled in centralized or decentralized manner. This paper presents and discusses exemplary use cases for our EOM that illustrate the centralized as well as the decentralized use of our approach within hybrid energy systems. Overall, we believe that the EOM represents the key approach for a further systematic development of an open hybrid energy grid.
\end{abstract}

\section{INTRODUCTION}

$\mathrm{T}$ HE tendency towards decentralized controlled energy conversion systems and the increasing number of ITenriched smart systems in general leads towards an energy landscape that consists of complex, globally connected and mainly software driven systems. In order to reach climate targets or just to maximize organizational profit, smart markets need to be provided on top of the underlying technical systems with their inherent flexibility. Concurrently, a stable volatile energy production must be guaranteed. However, global goals, such as the stabilization of a distribution network, require a minimum of adaptive interoperability that has to be expressed in one standard. We believe that the developments in smart grids and related areas are now at a point, were it has to be asked, if we want to build control systems that will create new monopolies, caused by proprietary software solutions, or if we want to support an unbundled and open energy supply that, on the one hand, offers the needed intelligent flexibility and, on the other hand, supports further developments over the next decades? Assuming that the latter is the case, it is obvious that software standards are required that prevent our already highly complex energy supply from becoming more complex and possibly uncontrollable with respect to the technical foundations and market regulations [1].

This paper presents the approach and the framework of our system-centered Energy Option Model (EOM), that permits to comprehensively describe the energetic and the economic behavior of any type of energy conversion system. Additionally, based on this unifying concept, the dynamic aggregation of hybrid energy systems is supported so that coalitions of systems, like virtual power plants, prosumers in a distribution network or even the devices in a Smart House can be represented, observed and optimally controlled. As a consequence of this unifying approach, the EOM can be used for several purposes and in different scenarios. By describing energy systems and their abilities in a comprehensive manner, the EOM can first be used as foundation for complex system simulations. Moreover, connected to real on-site systems, it permits the construction of centralized, partially decentralized or completely decentralized control approaches within Smart Grid and Smart Market scenarios. To demonstrate this capability, we demonstrate here two simple application cases by means of single and aggregated electrical vehicles. Further, we will discuss the consequences for our energy agent approach [2]. For this the paper is structured as follows: The next section provides some background information and motivates our work. Section III will outline the energy agent approach, while IV introduces the basic structure of the EOM. In section $\mathrm{V}$ the two mentioned use cases of the EOM will be shown. Subsequently, our solution will be discussed and compared to other approaches, known from literature. The paper end with a conclusion in section VI.

\section{BACKGROUNG \& MOTIVATION}

The ongoing discussions of whether Smart Grid devices or even more complex system aggregations are controlled in a centralized or decentralized manner and under which 
market rules they operate, is still an open scientific, organizational and social question. Here, the requirements or goals for such control approaches differ and may address stability issues in distribution networks, the efficient usage of energy resources, a cost and revenue optimization with respect to the actual part of the energy market or simply aspects of a customer's comfort. Based on these different requirements, diverse control solutions were developed. Accordingly, there is a lack of standardized control approaches that provide both, first an investment protection for developer and customer of smart devices and secondly an adaptive flexibility with respect to an organizational affiliation and thus to the actual control solution used with such devices. We will discuss these two aspects in the following.

In the context of control approaches, literature of the recent years presents a significant number of publications, describing the successful applications of agents and MultiAgent systems (MAS) in specific Smart Grid scenarios, as for example in virtual power plants [3], in Demand-SideManagement systems [4] or within price-based, indirect controlled approaches that are known as Demand Response [5]. Beyond, a few decentralized control approaches were introduced, as for example with [6] and other. Since most of these developments were motivated by the problems that occurred with the increasing number of regenerative and thus volatile energy production systems, they basically focus on aspects around electrical grids and markets. However, such a one-sided focus neglects the flexibility-potentials that could possibly be utilized in order to close the storage gap electrical networks. Research has already introduced a couple of approaches here that are characterized as so called Power-to-X applications [7]. In such applications, electrical excess energy will either be stored, as for example in an electrical vehicles accumulator (Power-to-Vehicle), or it is converted to a different form of energy, such as hydrogen or heat. In this context, [8] provides an overview of different approaches, technologies and strategies that focuses on the management of large-scale schemes of variable renewable electricity. Considerations about such hybrid energy systems, however, are fairly new in a broader range and thus even more far away from any standardization for an open and adaptive control.

The lessons that could be learned with the current state of the art approaches, clearly indicate that any further diversification of control approaches has to be prevented in order to avoid uncontrollable situations that may lead to a chaotic overall system behavior of our energy supply. Additionally, it can be expected that an increasing diversification of self-containing control approaches will also increase the customer's uncertainty about the expectable functionalities, its benefits, the customers privacy and will thus probably prevent the needed investment decisions that would bring the "smart" market into motion [9]. This similarly applies also for the developer and provider of such control solutions, since they have to live with the uncertainty of non-existing or suddenly appearing policies that may destroy their control approach or even their business models.

In contrast to an adaptive control that is know from automation [10], we are using the notion of an adaptive control as a synonym for an open overall system architecture that dynamically allows to adapt and thus to 'understand' any kind of energy conversion process and its inherent flexibility. Here we go by the claim that individual systems, or energy conversion processes respectively, should be arbitrarily integrated into any larger overall system. The reasons for this requirement are versatile. The simplest argument for that is the avoidance of new regional or local monopolies that occur, if proprietary and self-containing onsite solutions are used, as already mentioned in the introduction of this paper. Following the unbundling principle, it is our opinion that a customer should be able to freely select and change its energy supplier, regardless of what specific smart grid device or what actual control approach is used; this freedom must not be prevented by any smart device or software system.

The described bilateral relation between a customer and a supplier, however, does not go far enough, since the number of parties involved in this context is much larger. For example, it is conceivable that the system immanent flexibility of an energy conversion process can also be used by a distribution network operator (DNO) in order to stabilize critical network situations. But this would require that also a DNO is able to utilize a systems flexibility, which is not possible if no unified description of an energy conversion process is used.

In turn, if one sees a unified system description not only as a technical or regulatory requirement, such description would have the potential to be the key enabler for a real sustainable development of a market driven future energy grid. Starting from the scientific point of view, unified system descriptions could be used in order to systematically explore the complexity of large-scale system aggregations and their cross associations, as they can already be found in a single distribution network that is organizationally also connected to different energy suppliers. Using the same base control model for single systems, researchers could get the chance to similarly compute and compare their results and thus their different control approaches. Based on that, the necessary market rules could be derived and thus hopefully help to resolve the current chicken and egg problem in sustainably designing a future energy grid.

Based on that, also the energy market could participate in various ways. Providing an adaptive operation and flexibility model for any energy conversion process involved, could theoretically enable new market mechanisms that would for example allow to buy the needed, task-dependent energy for a single system on the fly. Assuming a suitable accuracy of such model, it could 
also be used as a predictive model that provides the information for the needed energy amount. Beyond that, also the change of supplier could be made easier, since the inclusion of a specific system would be based on the same operation and flexibility model.

With above described requirements or even visions, the here introduced EOM was firstly developed as a modular approach, focusing on the abilities and thus also on the flexibility of single energy conversion processes. This modularity concept goes along with our energy agent approach that will shortly be outlined in the next section. Since these energy agents may need to consider multiple systems simultaneously, the EOM and its framework were designed also to manage system aggregations. These can be understood as 'system of systems' and will be explained in more detail in section IV.

\section{ENERGY AGENTS}

According to our previous publication [2], an energy agent can be understood as the computing entity that manages the concerns between an on-site system and its stakeholder. For this, we see an energy agent as a mediator software system that for the most part is located in between of the local system controller and the outside, possibly "smart" world. An exception is the case, if the actual system being guided through an agent has no own controller; in this case an energy agent may also take the control tasks.

For the interaction with the local system, energy agents need to incorporate a multitude of possible connectivity protocols, as for example serial protocols like Modbus RTU or more sophisticated protocols like OPC UA, IEC 61850 and other [11], [12]. By using this connectivity, the energy agent should be enabled to receive or set all relevant information that help to comprehensively monitor or guide the actual system. In the context of the EOM, those information are part of the so called 'system variables' and include measurement values, system set points that can be controlled by the agent and system set points that were configured by an end user, as for example the desired room temperature for a heating system. To cooperate with further agents and software systems, as for example with a web service that provides weather forecasts, the energy agent should be able to use a network connection that allows these interactions. Fig. 1 below visualizes the described system environment and shows the full list of system variable types that are used within the EOM, described in the next section.

With the static information, system specific data models are meant that describe the system abilities in a mathematical, empirical or theoretical manner. Examples can be found with a consumption map of a combustion engine or with a turbine or compressor map of rotating equipment.

The remit of an energy agent depends on the actual scenario definition for which it will be designed. Here we introduced the notion of 'integration level' (IL) that describes the level of sophistication with respect to a scenario and thus also to the inherent complexity and the abilities of an energy agent [13]. In a rough classification, we differentiated between an IL0 that describes the initial construction state of the energy infrastructure for a time, where no decentralized computational entities could be found. Based on that and with increasing and coherent integration level, we assumed a continuous change from centralized controlled system to more and more decentralized controlled systems. Finally, we assumed that a completely decentralized control approach that was described with IL5 is only a theoretical consideration that can't be realized.

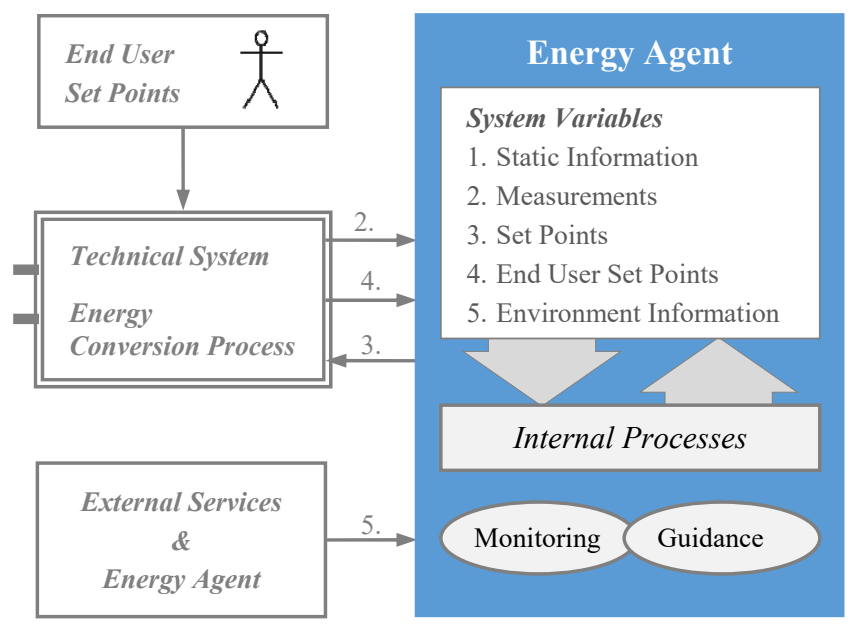

Fig. 1 Energy Agent Connectivity and its System Variables

With the coherent set of integration levels, we also wanted to reflect the fact that local software components as the energy agents will be subject to changing conditions over time. For example, it can be assumed that the regulatory policies will change successively over the next decades. Accordingly, the on-site software has to be further developed and adjusted in order to meet the changing market rules and policies. This, in fact, corresponds to another type of hybrid consideration, since it can be expected that not every system involved will always be updated.

It is our goal to use energy agent in simulations, testbed applications and in real on-site systems across all integration levels. Therefore, we are using the characteristics of software agents that allow to further enrich or exchange specific behaviors. Especially the ability to interact with the underlying technical system and thus to monitor or guide it, represents here the main difference between simulation, testbed or real application. Here it is planned, to either use a simulated or a real input and output behavior for the (virtual) connection to the technical system. However, while in a real system real data can be gathered, in simulations a mechanism is required that allows to simulate the acquisition data that would originally come from the underlying system. Additionally, these data have to dynamically change in case 
that an energy agent meets a control decision. That in turn requires a suitable model that allows to describe the dynamic system behavior.

It is obvious that an energy agent requires a suitable model and thus an understanding about the associated technical system for both, for simulations as well as for real applications. This applies not only if a decentralized control solution is to be used or validated, as for example in case of a price-based Demand Response approach. This already applies for the lowest integration level 0 , where basically the physical behavior of one or more energy conversion systems is described. Beyond, using a larger number of such dynamic models will further enable to simulate complex infrastructures, such as distribution or transportation networks and their dynamics.

Independent of the here introduced idea of an energy agent, we would like to state that an open description of the capabilities and hence the flexibilities of individual energy conversion systems is essential for a sustainable development of our energy infrastructure. Our approach is presented in the next section.

\section{THE ENERGY OPTION MODEL (EOM)}

The fundament for the EOM is a system delimitation that separates the considered energy conversion process (ECP) and its environment. Based on that, a customizable and scalable system definition can individually be modeled. That means that on the one hand small single systems can be described, while on the other hand also bigger systems or multiple simultaneous systems might be modeled at once, in case that the systems effect on the environment (or on the network connections respectively) can reliably be described. Thus, a system definition described by the EOM, can also be the model of a complex plant, even if it consists of several conversion processes in detail.

Based on the first law of thermodynamics [14], that describes the conservation of energy, the EOM allows to capture all network connections and usage types of an ECP. Therefore, the concept of a so called TechnicalInterfaceConfiguration (TIC) was introduced as an anchor for the further modelling that enables to differentiate those cases. Thus the EOM allows to capture different connectivity types (as for electrical vehicles) or to record different execution modes for single systems (as for example the different programs of a washing machine). Each TIC can be further modeled as described in the following.

For the further inventory of an ECP, the EOM allows to capture the actual network connections of the system. For a comprehensive description, the EOM requires information about the used energy carrier with each connection (e.g. electricity, natural gas or heat) and the direction of the energy flow, if relevant. In case that the described system contains an internal energy storage, additionally the storage capacity can be captured with a specific network connection.
To capture the regular operating states and thus the usual system behavior, the EOM allows their modelling with the help of a directed graph $G(V, E)$. Here, the vertices $V$ represent the operating states, while the directed edges $E$ describe the subsequent order in which the operating states occur. Through a reference to itself, an operating state can be repeated as many times as needed. Each vertex contains further information about the duration and the energy revenues that can be generated in an operating state. Defining the duration of an operating state and repeating it, allows thus a flexible modelling that corresponds to any desired discretization for the behavior of the considered ECP.

The description of an energy flow, in a specific operating state, for a specific network connection and for the chosen duration is a further part of the operating state description that corresponds to one of the above described vertices. The EOM offers various possibilities to describe energy flows. For that purpose, it differentiates between constant, empirical or calculated energy flows that can be used as description for each single network interface. Here, measurements that were statistically evaluated are seen as empirical information. Those data can especially be used in case that an energy flow is not simply constant or if the energy flow can't be calculated by mathematical equations. Thus unsteady or transient operating states can be described by datasets and help to better 'understand' or predict a systems behavior.

For the calculation of energy flows, the framework of the EOM offers the extension of specific classes which then contain the required calculation methods. Based on an operating state and the system variables that were already described in the previous section, any kind of calculation can be executed in order to determine an energy flow. Thus, this adaptive approach enables to use the theoretical knowledge that is especially present in the engineering disciplines.

Up to here, we described the first part of the EOM and its framework, which can be considered as the basic model and thus the fundamental for all further steps. The corresponding data structure of the above described model is also available as an XML scheme [2]. Based on that, the EOM-framework allows the persistence and thus the exchange of ECP descriptions.

The second part of EOM and its framework is aimed to comprehensively generate schedules for the system considered. Comprehensively means here that with the help of the EOM a full description of the system behavior over time can be generated that consist of all time-dependent values of the system variables and all applied energy flows. Furthermore, the converted energy amounts, the energy losses and an individual utility function can be taken into account, which enables an assessment of single system states and thus the assessment of the overall system usage. 
To get there, the EOM framework enables to configure the needed evaluation parameters that are first a starting, time-dependent system state and an end time, wherein the evaluation may be terminated. If required, the end time for an evaluation can also be connected to a target system state. This could be for example a fully charged battery of an electric vehicles battery at the end of the evaluation period.

Further information for the time-dependent evaluation of the system can be predictions of specific system variables that are required to calculate energy flows. Such variables can be, for example, temperature or insolation information that are derived from weather forecasts and that are used in order to pre-calculate the heat demand of a house or to predict the energy production of a photovoltaic plant. Here, the framework of the EOM permits again the extension and usage of individual implementations that may provide the needed data by using any type of data source.

For the definition of an individual utility function, the EOM separates between two assessment paths. The first path is designed to enable the assessment of the energy flows and amounts that are transferred over each network interface. Therefore, a corresponding function can be assigned for each energy carrier in combination to a flow direction. Thus, a power feed or consumption can be considered differently. With the second path of the utility function, the EOM enables once more the extension and usage of individual calculation classes. Within such a class, any type of calculation can freely be implemented; its result will finally be added to the utility results of the energy flow assessment. In this way, e.g. depreciation costs for the operation of an ECP can additionally be added or further, more complex relationships.

The settings described above, finalize the preparation of an evaluation process within the EOM framework and an actual evaluation process can be executed. Since the range of goals that have to be considered here may differ, the EOM framework permits once more to define individual so called 'evaluation strategies'.

Based on the information of the basic model and the evaluations settings, the goal of such a strategy is to produce a system schedule in a predefined data structure. Therefore, the EOM does not restrict the way, nor limits it to a specific algorithm that has to be used. Rather, it leaves the actual approach for the creation of a system schedule deliberately open in order to enable the application of different competitive approaches. That means that a developer is free to decide which algorithm is to be used and how the actual evaluation strategy is designed. This especially enables to reuse already proven and reliable approaches for the generation of system dependent schedules.

Beside the above described open architecture, the EOM framework additionally provides a comprehensive assistance for generating system schedules through an own evaluation strategy. Therefore, a graph based methodology was developed that theoretically can be applied to any kind of
ECP. The foundation for this approach is the unique identification of systems states. Since the identification of these states may differ depending on the actual system, the EOM permits to specify the parameters that have to be considered here. By default, the evaluation time, the chosen interface configuration, the operating state, the set points as well as controlled measurements are used in order to specify this identifier. Additionally, also storage loads can be taken into account, if required. For the discretization of unique system states, an increment can be set for each parameter of the state identifier.

Based on those clearly differentiable and discretized unique system states, a graph can be drawn that represents the states and their subsequent states over time. Figure 2 below shows the so called Differences Graph of this evaluation approach.

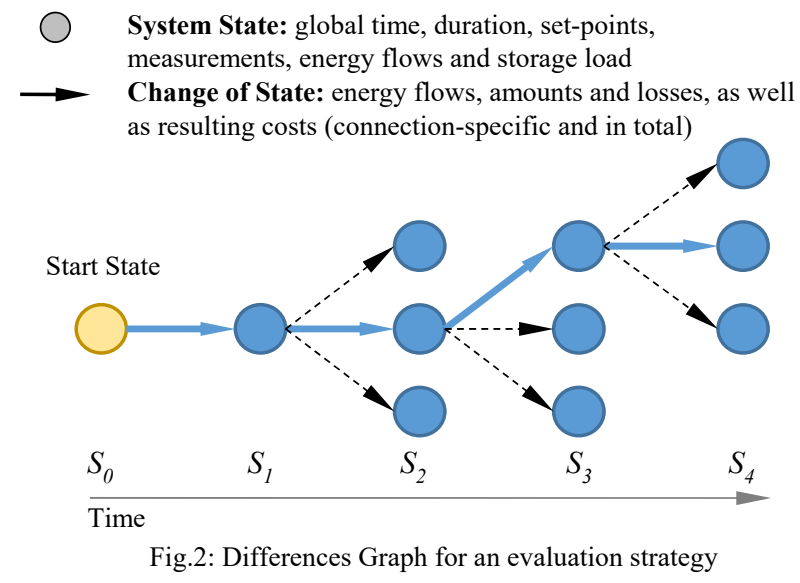

While the vertices of the graph represent the unique system states, the edges correspond to the actual changes that occur with the transition from one state to another. Thus, a change in a storage level can be determined as well as the changes that occur if an individual utility function is applied. Based on this differences approach, a number of well know graph-based optimization algorithms may be applied (e.g. Dijkstra and many more) in order to optimize the system usage, taking into account certain objectives. Additionally, the EOM framework provides a general algorithm structure that can be used within an evaluation strategy and that proceeds along the graph structure. For reasons of space we skip a detailed explanation here, but we want to point out that an evaluation becomes a sequence of time dependent decision processes that are based on qualified system information, represented by the Differences Graph. Thus, the EOM and its framework may also be considered as a decision support system [15].

The aggregation method for several technical systems that is provided by the EOM is realized in the sense of a 'system of systems'. Analogously to a single technical system, the aggregator will also be considered as a technical system, summarizing the sub systems in regard to interfaces by energy carrier and by add up energy carrier-dependent 
storage capacities. In more complex scenarios, where wider areas are to be considered, an aggregation may additionally require a network calculation, which is also supported by the EOM. Similar to the evaluation strategy for single technical systems, a strategy for aggregated technical systems can individually be designed. In contrast to single systems, decisions must be made for each subordinate system, so that several decision graphs will concurrently be used during an evaluation of aggregated ECP's.

The EOM is available as an end user application and laboratory tool, but can also be used "head-less" within an energy agent. In this case it is the task of an agent to get and provide the needed base information for a single technical system or an aggregation of technical systems (e.g. predictions, cost information and other) and start an appropriate evaluation process, if required.

\section{APPLICATION OF THE EOM}

To demonstrate the applicability of the EOM, this section describes the modelling and algorithm approach for planning processes of one and more electrical vehicle's (EV) battery. For this, first, the actual system will be described in the next sub-section, while subsequently the used algorithms will be outlined.

\section{A. Single Electrical Vehicle}

The chosen EV has a battery with a storage capacity of 24 kWh. According to IEC 62196, it can differently be connected to an electrical network. For the experiments we have chosen a Mode 1 connection, which corresponds to a slow charging from a household-type socket-outlet $(230 \mathrm{~V}$, $3.5 \mathrm{~kW}$ charging or discharging). With this information, the base graph of the operating states was modelled as shown in Figure 3.

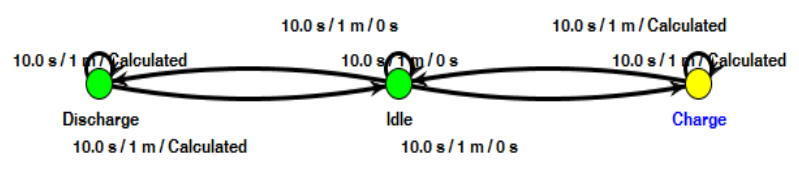

Fig.3: Graph for the operating states of an EV battery

It can be seen that the operation states Charge and Discharge are modelled in a way that each of them apply for at least 10 seconds. Both times are followed by a minimum and a maximum time in which the system can be kept in the respective operating state. While here the minimum time was defined in order to avoid a too short stay and thus a too quick change of the operating state, the maximum time can only be calculated depending on the current storage load (e.g. a full battery cannot be charged anymore). Further, for the operating state Idle no maximum must be defined, since this system neutral state has no time limit in principal.

For the evaluation of the system, we assumed a planning scenario, where the EV has a time frame between 8 p.m. and 6 a.m. for charging. Further, we assumed an initial storage load of $5 \mathrm{kWh}$, while the car has to be fully charged in the morning. These assumptions correspond to the definition of two system states (an initial and an end system state) with corresponding timestamps. This limits the evaluation period for the developed evaluation strategies. For the assessment of the time dependent system behavior, we used the EOM's cost function approach by means a time variable pricing for the usage of electrical energy.

To create a base for later comparisons, we first developed a simple strategy that's behaves in a regular or Greedystyled manner. That means, like a non-controlled or scheduled charging process, the EV's battery will directly be charged until it reaches its storage capacity.

Thereon based, another strategy was developed that considers the specified procurement costs for electrical energy over time. Simply by selecting time ranges that have the best prices and put them in an ascending order, the prioritized charging times were determined. Consequently, for the used evaluation strategy, the task of cost optimization results to a fairly simple observation of time and a selection of the desired operating state until the battery reaches it maximum capacity. Figure 4 below shows the used electricity costs and compares the results of the two evaluation strategies.
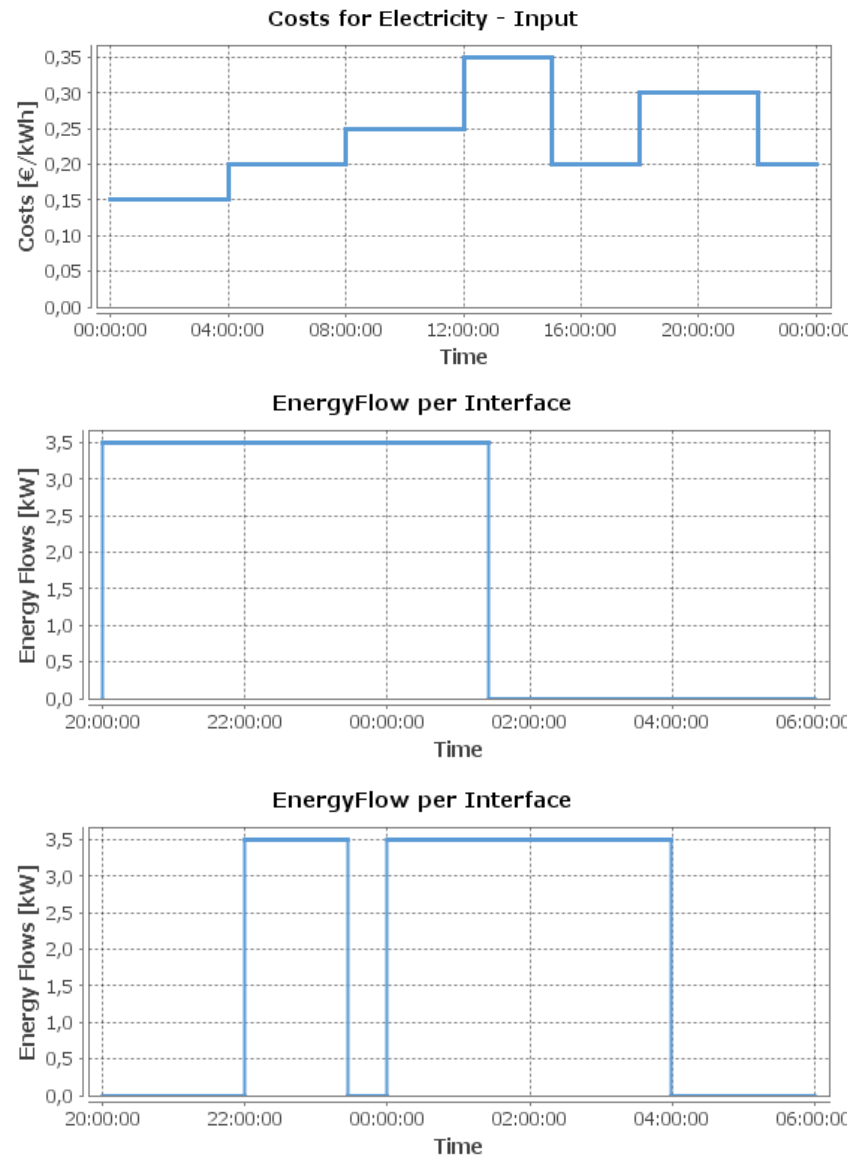

Fig.4: Cost-Optimal Charging of an EV's battery, based on price signals

For the example, a saving of $27 \%$ of the procurement costs could already be realized, simply by the described more sophisticated selection process of charging times. 
Even the complexity of the used algorithms, or evaluation strategies respectively, were very manageable here, the learning curve for the development of the EOM and its usage as a reasoning model were remarkable. Here we realized that, as first, the development of an evaluation strategy is required that is able to reproduce the regular behavior of an ECP. Only on this basis, the improvements of 'intelligent' algorithms can be found and compared.

Despite the simplicity of the presented example, a very important capability of the EOM is presented here. This is the capability to concurrently apply different evaluation or plan generation strategies in a parallel manner. Since competitive evaluation strategies can be executed concurrently, single energy agents can be empowered to become intelligent agents. Based on the current system state that can be acquired from the system (by means of measured values, set-points, energy flows etc.) and a defined goal, like a cost optimal charging of an EV's battery, the energy agent can execute different strategies, where each results to a different system usage of time. Based on a comparison or assessment of these resulting execution schedules, the energy agent is able to select the best plan and consequently to optimize the systems behavior. With this general approach, the EOM is closely related to the well-known BDI-concept [16], but its focusses on the special needs of the energy domain.

\section{B. Multiple Electrical Vehicles}

For the second application case of the EOM, we used the capability to consider several systems within an EOMaggregation. For this, the EOM supports the dynamic configuration of aggregations by means a tree organized structure, where the root node represents the overall aggregation and the sub nodes the aggregated systems. Analogously to single systems, the aggregation can be modeled as a system, while the considered sub systems can either be defined as static load curves, as dynamic single systems or as dynamic aggregations again. The base model of the aggregation was defined as shown in Figure 5.

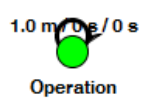

Fig.5: Graph for the operating state of an EV Fleet Aggregation

It can be recognized that the superior system of the aggregation is specified as system with a single operation state Operation, where the discrete time step is defined with 1 minute. Further, neither a minimum nor a maximum time is defined.

For the experiments, we used the above described EV model and varied the number of systems between $5,10,20$, 30, 40, 50 75, 100, 150 and 200 EV's. Since a price optimal charging for the whole EV fleet results to a similar curve in terms of the time course (e.g. 10 EV's, charging by $3,5 \mathrm{~kW}$ $=35 \mathrm{~kW}$ ), we slightly changed the objective of the evaluation strategy. In addition to a possible price optimal charging, an upper bound for the overall energy consumption should not be exceed now.

To archive this goal, the developed strategy first determined the demand of energy for the evaluation period. Further, it again sorted the consumption costs in an ascending manner. Subsequently, and with respect to the maximal available power that was defined by the upper boundary, the needed energy amounts could successively be assigned to the sub systems. Figure 6 below shows the resulting energy consumption for an EV fleet, consisting of 10 cars, where the upper bound was defined with $21 \mathrm{~kW}$.

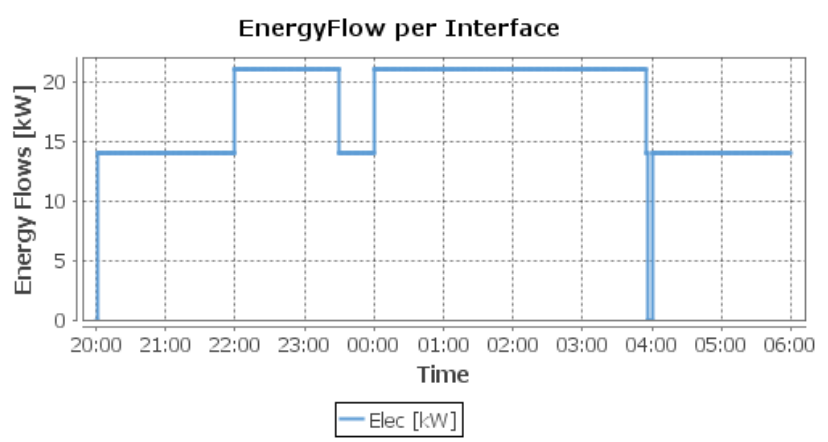

Fig.6: Cost-optimal charging with upper boundary for an EV Fleet

Compared to a simple price-optimal charging without upper boundary, the resulting costs were naturally slightly higher, since energy had to be consumed during periods with higher prices.

The time for the execution of the evaluation strategy and the creation of the execution schedules for each sub system ranged between 0.13 seconds for a single and 37.7 seconds for 200 systems. Figure 7 below shows the determined relationship between the number of systems involved and the thereon depending time for the evaluation process.

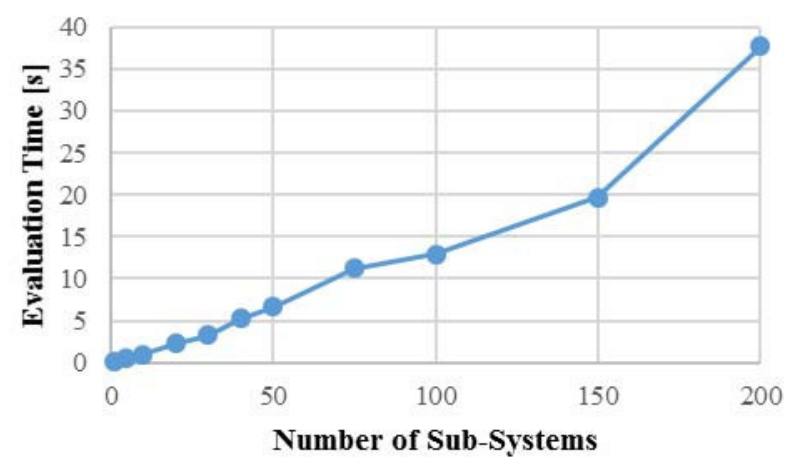

Fig.7: Time consumption for different numbers of sub systems

Since we considered the usage of the EOM within a planning process here, the required times for the evaluations seem to be very promising for us.

In addition to the experiments shown here, further application scenarios for the EOM were already investigated. Here we tested and improved the EOM for the usage with different energy carrier, but also for the real time 
application within an electrical distribution network. For reasons of space, unfortunately, these experiments and results can not be explained or shown in this paper and will be part of future publications.

\section{DISCUSSION \& COMPARISONS}

Starting from the modular perspective, we already mentioned the use of the EOM within an energy agent that is located within or beside an on-site ECP. Here it is the task of the agent to configure the setup for an evaluation as described in the previous sections. Since the EOM also enables the parallel execution of several evaluation strategies, the final result may consist of a number of alternative schedules for the underlying system. As a next step, one plan has to be selected and passed into an execution process. This process then has to care about the system execution according to the selected plan. With the ability to deliberatively provide several plans for an agent, the EOM can also be considered as a kind of BDI concept that is well known in the agent community. In contrast to the general BDI concept the EOM, however, is concretely designed to meet the needs for comprehensively consider hybrid ECP's.

Furthermore, the EOM concurrently provides the ability to consider several ECP's within a system aggregation. This additionally enables to create central control approaches as they are realized in a vast majority today, e.g. in virtual power plants. Assuming the availability of suitable system descriptions, with the help of the EOM, adaptive mechanisms could be realized that enable a flexible integration of any type of ECP. Here the only question is, who initially owns the EOM; in case that this knowledge is located on-site, the model has to be transferred to the aggregator. Analogously to an on-site located energy agent, it is the task of the centralized aggregator process to collect all needed information for the Sub-ECP's and execute one or more evaluations strategies for the aggregation, in order to determine and select the overall and the sub-schedules.

But also for completely decentralized control approaches, the EOM can be applied. Therefore, a sequential turn-based message process can be considered. Instead of the use of the EOM for single systems, however, each system uses the aggregation part of the EOM. Here, the aggregation basically consists of the single local system and a summarizing schedule of all previous systems. The task of the local evaluation process is thus to generate a new local and a summarizing schedule. The latter has to be forwarded to the next ECP or energy agent respectively.

In addition to the above described three application types (local, centralized and decentralized), we believe that further types can be derived combinatorically. For example, several decentralized control processes could be controlled centrally and so on. In our view the EOM can be used for planning as well as for a real time control purposes. A crucial point, however, is the temporal resolution with which processes are modeled. While a fine granular resolution is helpful for real time applications, it will make planning process more expensive in the sense of needed computing time: every discrete step will require to resolve all possible subsequent states and thus to meet a decision for it. We assume that the modelling also requires to find a trade-off for that, but we can't answer this question at this point. Therefore, we will investigate the application of the EOM for further real time process in the near future.

Comparing the EOM with other approaches, requires first to highlight its unique position. Those highlights can be found with several facets that are: a) the comprehensive, system centric consideration of an energy conversion process that enables local reasoning processes for intelligent energy agents, b) the non-exclusive consideration of electrical energy and c) the adaptive characteristic that is offered by the EOM and that enables to realize centralized as well as decentralized control approaches.

Starting with the latter aspect and focusing on centralized control approaches, it is clear that such control approaches are in the main focus of most energy provider currently. Concrete solutions like this are known as energy management systems or can be found with the approach of the demand side management that was already mentioned in the background section of this paper. Here, one well known solution can be found e.g. with the PowerMatcher [17] and other. Beyond, also examples for decentralized control approaches were referred in the background section, but compared to the EOM, no solution known to us provides such flexibility for the actual implementation of a concrete control approach (e.g. centralized and/or decentralized).

Further, the consideration of energy conversion systems in general is a quite new aspect in the current Smart Grid research. Thus, similar adaptive and hybrid approaches as they are provided by the EOM are new and could not be found yet. Several general structures for an IT-based, interoperable management of distributed system are provided by the Common Information Model (CIM). Since the organizing Distributed Management Task Force ${ }^{l}$ basically combines the interests of IT organizations and companies, energy specific model definitions like the EOM can't be found here.

Last but not least, the reasoning capability that is provided by the possible parallel execution of different evaluation strategies is a very unique property of the EOM. Even this idea is similar to the general BDI concept, the EOM is the only known, energy specific approach that provides the needed description cardinality for hybrid energy conversion processes. Thus, it has the capability to meet the broad range of requirements and applications in the energy domain.

\footnotetext{
${ }^{1}$ http://www.dmtf.org/
} 


\section{CONCLUSION \& OUTLOOK}

In this paper we presented our concept and the control approach for hybrid energy conversion processes by means a unifying Energy Option Model (EOM). Since this model has the capability to describe all relevant types of energy conversion processes, it provides the foundation for the design of new adaptive control approaches that permit a dynamic aggregation and optimization of aggregations of energy conversion processes.

In the next years, the model will be improved and further developed under the project Agent.HyGrid. Here it is planned to close the gap between agent-based simulations and real-world applications in order to produce comparable results for both cases. Thus, a realistic laboratory and testbed environment will be created for control solution of Future Energy Grids.

Overall, we believe that standards that enable a homogeneous and in particular open development of "smart" energy systems are urgently required; for science as well as for systems used in real applications on-site. We further believe that therefore the concept of a generally accepted energy agent with a unified description of the underlying technical system that we call Energy Option Model is the necessary foundation.

The EOM introduced was registered for a patent. The grant is pending.

\section{REFERENCES}

[1] V. Vyatkin, G. Zhabelova, N. Higgins, M. Ulieru, K. Schwarz, and N. C. Nair, "Standards-enabled Smart Grid for the future Energy Web," in Innovative Smart Grid Technologies (ISGT), 2010, 2010, pp. 1-9.

[2] C. Derksen, T. Linnenberg, R. Unland, and A. Fay, "Structure and Classification of Unified Energy Agents as a base for the systematic development of Future Energy Grids," EAAI-Engineering Applications of Artificial Intelligence, 2014.
[3] D. Nestle and J. Ringelstein, "Integration of DER into Distribution Grid Operation and Decentralized Energy Management," Smart Grids Europe, vol. 19, 2009.

[4] [Online] Available at: http://www.e-energy.de/de/modellregionen.php

[5] A. Faruqui and S. Sergici, "Household response to dynamic pricing of electricity: a survey of 15 experiments," Journal of Regulatory Economics, vol. 38, no. 2, pp. 193-225, 2010.

[6] S. Vandael, N. Boucké, T. Holvoet, K. De Craemer, and G. Deconinck, "Decentralized Coordination of Plug-in Hybrid Vehicles for Imbalance Reduction in a Smart Grid," in The 10th International Conference on Autonomous Agents and Multiagent Systems - Volume 2, 2011, pp. 803-810.

[7] A. Sternberg and A. Bardow, "Power-to-What? - Environmental assessment of energy storage systems," Energy Environ. Sci., vol. 8, no. 2 , pp. $389-400,2015$.

[8] P. D. Lund, J. Lindgren, J. Mikkola, and J. Salpakari, "Review of energy system flexibility measures to enable high levels of variable renewable electricity," Renewable and Sustainable Energy Reviews, vol. 45, pp. 785-807, 2015.

[9] T. Krishnamurti, D. Schwartz, A. Davis, B. Fischhoff, W. B. de Bruin, L. Lave, and J. Wang, "Preparing for smart grid technologies: A behavioral decision research approach to understanding consumer expectations about smart meters," Energy Policy, vol. 41, pp. 790-797, 2012.

[10] P. A. Ioannou and J. Sun, Robust adaptive control. Courier Corporation, 2012.

[11] [Online] Available at: http://www.modbus.org.

[12] R. E. Mackiewicz, "Overview of IEC 61850 and Benefits," in Transmission and Distribution Conference and Exhibition, 2005/2006 IEEE PES, 2006, pp. 376-383.

[13] C. Derksen, T. Linnenberg, R. Unland, and A. Fay, "Unified Energy Agents as a Base for the Systematic Development of Future Energy Grids.," in MATES, 2013, vol. 8076, pp. 236-249.

[14] G. J. Van Wylen, R. E. Sonntag, and C. Borgnakke, Fundamentals of Classical Thermodynamics, no. Bd. 1. Wiley, 1994.

[15] D. J. Power, R. Sharda, and F. Burstein, Decision support systems. Wiley Online Library, 2002.

[16] J. Sudeikat, L. Braubach, A. Pokahr, W. Lamersdorf, and W. Renz, "Validation of BDI Agents," in PROMAS, 2006, pp. 185-200.

[17] J. K. Kok, C. J. Warmer, and I. G. Kamphuis, "PowerMatcher: Multiagent Control in the Electricity Infrastructure," in Proceedings of the Fourth International Joint Conference on Autonomous Agents and Multiagent Systems, 2005, pp. 75-82. 